\title{
Preço mínimo de venda da madeira de eucalipto para os segmentos de carvão e serraria
}

\author{
Luis Carlos de FREITAS ${ }^{1}$, Wilmerson Bernadino PRADO ${ }^{1}$, Aline Pereira das VIRGENS ${ }^{1 *}$, \\ Danusia Silva LUZ ${ }^{1}$, Ana Carolina Dantas MOREIRA ${ }^{2}$
}

${ }^{1}$ Universidade Estadual do Sudoeste da Bahia, Vitória da Conquista, BA, Brasil.

*E-mail: apereira.aline@hotmail.com

Recebido em julho/2017; Aceito em maio/2018.

RESUMO: Objetivou-se neste trabalho avaliar o preço mínimo de venda de madeira de eucalipto com reflorestamentos destinados aos segmentos de carvão e serraria. O preço mínimo foi determinado através dos dados de custos e receitas dos empreendimentos. Os custos dos projetos foram descontados para o ano zero, somados e igualados a receita, também descontada para o ano zero. A receita foi expressa pelo produto do Incremento Médio Anual, idade de corte, e preço mínimo de venda da madeira. Foram obtidos os seguintes preços mínimos de venda para madeira: carvão ( $\mathrm{R} \$ 38,44$ para madeira em pé e $\mathrm{R} \$ 55,39$ para madeira empilhada); serraria ( $R$ \$ 29,76 para madeira em pé e $\mathrm{R} \$ 57,71$ para madeira empilhada). Os valores encontrados foram inferiores ao preço de mercado, evidenciando que o custo de produção da madeira foi totalmente assegurado pelo seu preço de venda, proporcionando ainda um adicional de receita para cada metro cúbico comercializado. A análise de sensibilidade mostrou grande influência da taxa de juros no preço mínimo de venda da madeira, diferentemente do que foi observado para o custo anual de arrendamento da terra. Os resultados da pesquisa mostram importância ao nortear produtores florestais em relação aos preços de comercialização de produtos.

Palavras-chave: planejamento econômico, projetos florestais, custo de madeira.

\section{Minimum sale price of eucalypt timber considering reforestation to vegetal coal production and sawmill}

\begin{abstract}
The objective of this study was to evaluate the minimum selling price of eucalyptus wood with reforestation for the coal and sawmill segments. The minimum price was determined by the cost and revenue data of the projects. Project costs were discounted for year zero, plus and equal to revenue, also discounted for year zero. Revenue was expressed as the product of the Annual Average Increase, age of cut, and minimum selling price of the wood. The following minimum selling prices were obtained for wood: coal ( $\mathrm{R} \$ 38.44$ for standing timber and R \$ 55.39 for stacked timber); ( R \$ 29.76 for standing timber and R \$ 57.71 for timber stacked). The values found were lower than the market price, evidencing that the cost of production of the wood was fully assured by its selling price, also providing an additional revenue for each cubic meter marketed. The sensitivity analysis showed a large influence of the interest rate on the minimum selling price of the wood, unlike what was observed for the annual cost of renting the land. The results of the survey show importance when guiding forest producers in relation to the prices of products commercialization.
\end{abstract}

Keywords: economic planning, forestry projects, timber cost.

\section{INTRODUÇÃO}

O setor florestal tem contribuído de forma positiva para o avanço da economia do país. No ano de 2016 a receita proveniente das exportações do setor brasileiro de árvores plantadas alavancou o equivalente a US\$ 8,9 bilhões, atingindo 6,2\% do PIB Industrial. O país é detentor de uma área de 7,84 milhões de hectares de reflorestamento sendo esse setor responsável por $91 \%$ de toda a madeira produzida para fins industriais. (IBÁ 2017).

A crescente expansão do consumo de madeira e dos seus derivados conduz à busca de análises econômicas mais consistentes e precisas de forma manter a competitividade e sustentabilidade do setor. Nesse contexto, destaca-se a importância de se avaliar o preço mínimo de venda da madeira. Esta variável consiste em um elemento chave e estratégico para o sucesso de projetos florestais, sendo fortemente influenciada pela duração dos horizontes de planejamento (ALMEIDA et al., 2010).

Para determinação do preço mínimo de venda da madeira deve-se analisar a cadeia produtiva do segmento, buscando determinar os custos nos processos de implantação, condução e colheita florestal. Os referidos custos descontados para o ano zero, quando somados e igualados a receita, também no ano zero, definem a equação base para determinação do preço mínimo de venda da madeira (SILVA et al., 2005).

A diferença entre o valor real de mercado e o preço mínimo determina o lucro líquido do empreendedor florestal, auxiliando o mesmo na gestão financeira e no planejamento de sua atividade. O preço da madeira consolida-se como uma incerteza econômica de alta volatilidade, sobretudo, pela distância de transporte (VALVERDE et al., 2004); condições de manejo (SCOLFORO, 2001) e pelas condições de oferta e demanda (ALMEIDA et al., 2009).

Pouco se sabe sobre o comportamento do preço mínimo de venda da madeira no estado da Bahia, o que poderá auxiliar os produtores da região no estabelecimento de suas margens de lucro, quando da comercialização de seus produtos. Análise projetada para determinação do preço mínimo de venda da madeira constitui, contudo em uma ferramenta de planejamento econômico capaz de nortear os empreendedores 
florestais em relação a uma tomada de preço mais segura, garantido a sustentabilidade ao setor.

No contexto da análise de sensibilidade, destaca-se a importância de se conhecer as variáveis mais influentes na composição do preço mínimo, o que pode auxiliar os produtores no manejo de custos envolvidos na cadeia produtiva. Assim, este trabalho teve como objetivo avaliar os preços mínimos de venda da madeira de eucalipto em dois empreendimentos florestais no estado da Bahia, bem como promover uma análise de sensibilidade com vista conFigurar um possível cenário para os respectivos preços em relação às variações nas taxas de juros e no custo anual da terra.

\section{MATERIAL E MÉTODOS}

2.1. Área de estudo e caracterização dos empreendimentos avaliados

Os dados da pesquisa foram obtidos em duas empresas do setor florestal, sendo uma produtora de madeira para o segmento de carvão vegetal (empresa 1) e a outra para serraria (empresa 2). A primeira está situada no município de Pojuca, no estado da Bahia, enquanto a segunda encontra-se inserida no município de Vitória da Conquista. De acordo com Köppen (1948), o clima onde a empresa 1 se situa é classificado como Af, com condições propícias de calor, umidade e precipitações, as quais são marcantes durante todo o ano, variando entre 1300 a $2300 \mathrm{~mm}$, sem estação seca. A empresa 2, de acordo com Novaes et al. (2008) apresenta clima tropical de altitude $(\mathrm{Cwb})$, considerado ameno devido a altitude, que apresenta em média 880 metros. A temperatura média anual é de $21^{\circ} \mathrm{C}$ e a precipitação varia entre 700 e $1100 \mathrm{~mm}$ anuais, distribuída nos meses de novembro a março, com um período seco de quatro a cinco meses, o solo da região corresponde ao Latossolo Amarelo Distrófico. A Tabela 1 ilustra as principais informações técnicas obtidas nos empreendimentos florestais avaliados.

Tabela 1. Variáveis técnicas dos empreendimentos florestais avaliados.

Table 1. Technical variables of the forest projects evaluated.

\begin{tabular}{lcc}
\hline \multicolumn{1}{c}{ Dados técnicos } & Empresa 1 & Empresa 2 \\
\hline IMA (m $\mathrm{m}^{3} /$ ha/ano) & 42,00 & 30,00 \\
Regime de manejo & Alto fuste & Alto fuste \\
Espaçamento padrão $(\mathrm{m})$ & $4,00 \times 2,25$ & $3,00 \times 3,00$ \\
Mudas & Clones & Sementes \\
Fator de empilhamento & 1,65 & - \\
Diâmetro $(\mathrm{cm})$ & 8 à 30 & $>12$ \\
\hline
\end{tabular}

IMA: Incremento Médio Anual; $\mathrm{m}^{3}$ : metro cúbico; ha: hectare; m: metro; cm: centímetro.

\subsection{Análises de custos e receitas}

Os custos relativos às etapas de implantação, manutenção e colheita florestal foram obtidos por hectare (ha). O custo anual de arrendamento $(\mathrm{Ca})$ foi determinado multiplicando o valor de um hectare de terra nua, nas regiões de ocorrência dos respectivos projetos, pela taxa mínima de atratividade anual (SCOLFORO et al, 2001). A Tabela 2 ilustra os custos de produção referentes aos projetos de reflorestamento com eucalipto.

Foram elaborados dois fluxos de caixa para os projetos avaliados sendo os custos por hectare, distribuídos nos seus respectivos anos de ocorrência dos horizontes de planejamento. As receitas foram expressas pelo produto do Incremento Médio Anual (IMA), idade de corte (em anos) e preço mínimo de venda da madeira, este último caracterizado como incógnita da pesquisa (FIGURAS 1e 2).

\subsection{Determinação do preço mínimo de venda da madeira}

A determinação do preço mínimo de venda da madeira em pé e empilhada baseou-se nos custos e receitas por hectare dos projetos avaliados. Os respectivos custos, descontados para o ano zero, foram somadas e igualadas as receitas, também no ano zero, conforme equações 1 e 3 , referente ao projeto de reflorestamento da empresa 1 e equações 2 e 4 , referente ao projeto de reflorestamento da empresa 2. A taxa mínima de atratividade considerada no estudo foi de $6,00 \%$ a.a ao ano. Vitale e Miranda (2010) utilizaram taxas de juros de 6,75\% a.a para análise comparativa de viabilidade econômica de plantios florestais, alegando que a respectiva taxa foi adotada no Programa de Plantio Comercial e Recuperação de Florestas (PROPFLORA), gerenciada pelo Banco Nacional de Desenvolvimento Econômico e Social (BNDES), vigente até 30/06/2009.

$$
\begin{aligned}
& \text { Custo ano } 0+\frac{\text { Custo ano } 1}{(1+\mathrm{i})^{1}}+\frac{\text { Custo ano } 2}{(1+\mathrm{i})^{2}}+\frac{\text { Custo ano } 3}{(1+\mathrm{i})^{3}}+ \\
& \frac{\text { Custo ano } 4}{(1+\mathrm{i})^{4}}+\frac{\text { Custo ano } 5}{(1+\mathrm{i})^{5}}+\frac{\text { Custo ano } 6}{(1+\mathrm{i})^{6}} \\
& +\frac{\mathrm{Ca} \times\left(1-(1+\mathrm{i})^{-6}\right)}{\mathrm{i}}=\frac{\text { Produtividade }}{(1+\mathrm{i})^{6}} \times \mathrm{Pl} \\
& \text { (Equação 1) } \\
& \text { Custo ano } 0+\frac{\text { Custo ano } 1}{(1+\mathrm{i})^{1}}+\frac{\text { Custo ano } 2}{(1+\mathrm{i})^{2}}+\frac{\text { Custo ano } 3}{(1+\mathrm{i})^{3}} \\
& +\frac{\text { Custo ano } 4}{(1+\mathrm{i})^{4}}+\frac{\text { Custo ano } 5}{(1+\mathrm{i})^{5}}+\frac{\text { Custo ano } 6}{(1+\mathrm{i})^{6}} \\
& +\frac{\text { Custo ano } 7}{(1+\mathrm{i})^{7}}+\frac{\mathrm{Ca} \times\left(1-(1+\mathrm{i})^{-7}\right)}{\mathrm{i}}=\frac{\text { Produtividade }}{(1+\mathrm{i})^{7}} \times \mathrm{Pl} \\
& \text { (Equação 2) }
\end{aligned}
$$

em que: $\mathrm{i}:=$ taxa anual de juros (decimal); Ca: custo anual da terra; Pl: preço líquido da madeira (madeira em pé).

$$
\begin{aligned}
& \text { Custo ano } 0+\frac{\text { Custo ano } 1}{(1+\mathrm{i})^{1}}+\frac{\text { Custo ano } 2}{(1+\mathrm{i})^{2}}+\frac{\text { Custo ano } 3}{(1+\mathrm{i})^{3}} \\
& +\frac{\text { Custo ano } 4}{(1+\mathrm{i})^{4}}+\frac{\text { Custo ano } 5}{(1+\mathrm{i})^{5}}+\frac{\text { Custo ano } 6}{(1+\mathrm{i})^{6}} \\
& +\frac{\text { Custo colheita }}{(1+\mathrm{i})^{6}}+\frac{\mathrm{Ca} \times\left(1-(1+\mathrm{i})^{-6}\right)}{\mathrm{i}}=\frac{\text { Produtividade }}{(1+\mathrm{i})^{6}} \times \mathrm{Pb} \\
& \text { (Equação 3) } \\
& \text { Custo ano } 0+\frac{\text { Custo ano } 1}{(1+\mathrm{i})^{1}}+\frac{\text { Custo ano } 2}{(1+\mathrm{i})^{2}}+\frac{\text { Custo ano } 3}{(1+\mathrm{i})^{3}} \\
& +\frac{\text { Custo ano } 4}{(1+\mathrm{i})^{4}}+\frac{\text { Custo ano } 5}{(1+\mathrm{i})^{5}}+\frac{\text { Custo ano } 6}{(1+\mathrm{i})^{6}} \\
& +\frac{\text { Custo ano } 7}{(1+\mathrm{i})^{7}}+\frac{\text { Custo colheita }}{(1+\mathrm{i})^{7}}+\frac{\left.\mathrm{Ca} \times 1-(1+\mathrm{i})^{-7}\right)}{\mathrm{i}}=\frac{\text { Produtividade }}{(1+\mathrm{i})^{7}} \times \mathrm{Pb} \\
& \text { (Equação 4) }
\end{aligned}
$$

em que: $\mathrm{i}=$ taxa anual de juros (decimal); $\mathrm{Ca}=$ custo anual da terra; $\mathrm{Pb}=$ preço bruto da madeira (madeira empilhada, considerando custo da colheita)

\subsection{Análise de sensibilidade}

Após determinação do preço mínimo de venda da madeira, realizou-se uma análise de sensibilidade tomando, como parâmetro, as variáveis taxa de juros e custo anual da terra. Considerou-se um acréscimo de $2 \%$ e $4 \%$ na taxa de juros utilizada $(6,00 \%$ a.a). Para o custo anual da terra projetou-se 
variações de $5 \%$ e $10 \%$ para mais e para menos nos valores adotados (Tabela 5).

\section{RESULTADOS}

A empresa 1 apresentou maiores custos nas etapas de implantação e manutenção, sendo o custo de implantação $22,34 \%$ superior em relação ao projeto de reflorestamento da empresa 2 (Tabela 2). Após determinação dos custos, os mesmos foram projetados nos seus respectivos horizontes de planejamento (Figuras 1 e 2).

Receita $=$ Produtividade $\mathrm{x}$ preço mínimo de venda da madeira

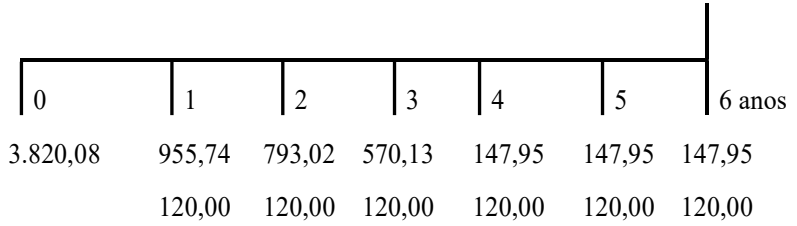

$4.273,31$

Figura 1. Fluxo de caixa referente ao reflorestamento com eucalipto (empresa 1).

Figure1. Cash flow referring to the reforestation with eucalyptus (company 1).
Receita $=$ Produtividade $\mathrm{x}$ preço mínimo de venda da madeira

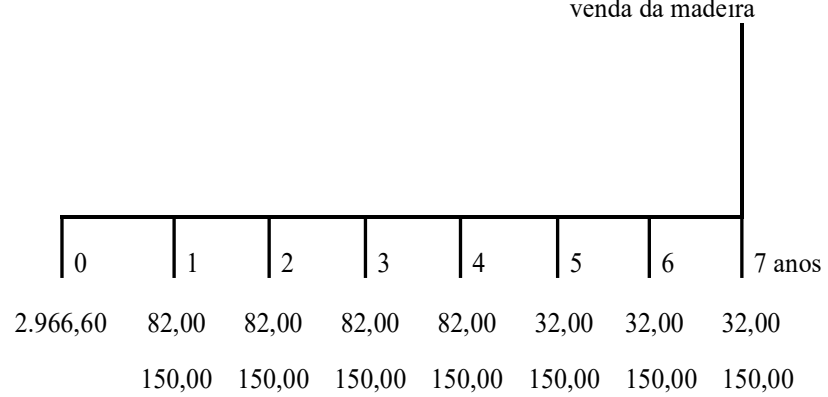

$5.870,00$

Figura 2. Fluxo de caixa referente ao reflorestamento com eucalipto (empresa 2).

Figure 2. Cash flow related to eucalypt reforestation (company2).

Os preços mínimos de venda da madeira em pé e empilhada encontram-se ilustrados na Tabela 3. Na empresa 1, o custo da colheita proporcionou um aumento de 16,95 reais no preço mínimo de venda da madeira, para cada metro cúbico comercializado. Este valor correspondeu a $30,60 \%$ do custo total de produção. Na empresa 2, a colheita foi responsável por um adicional de 27,95 reais, por metro cúbico, com impacto de 48,43\% no custo de produção (Tabela 3).

Tabela 2. Componentes de custos dos projetos florestais avaliados.

Table 2. Cost componentes of forest projects evaluated.

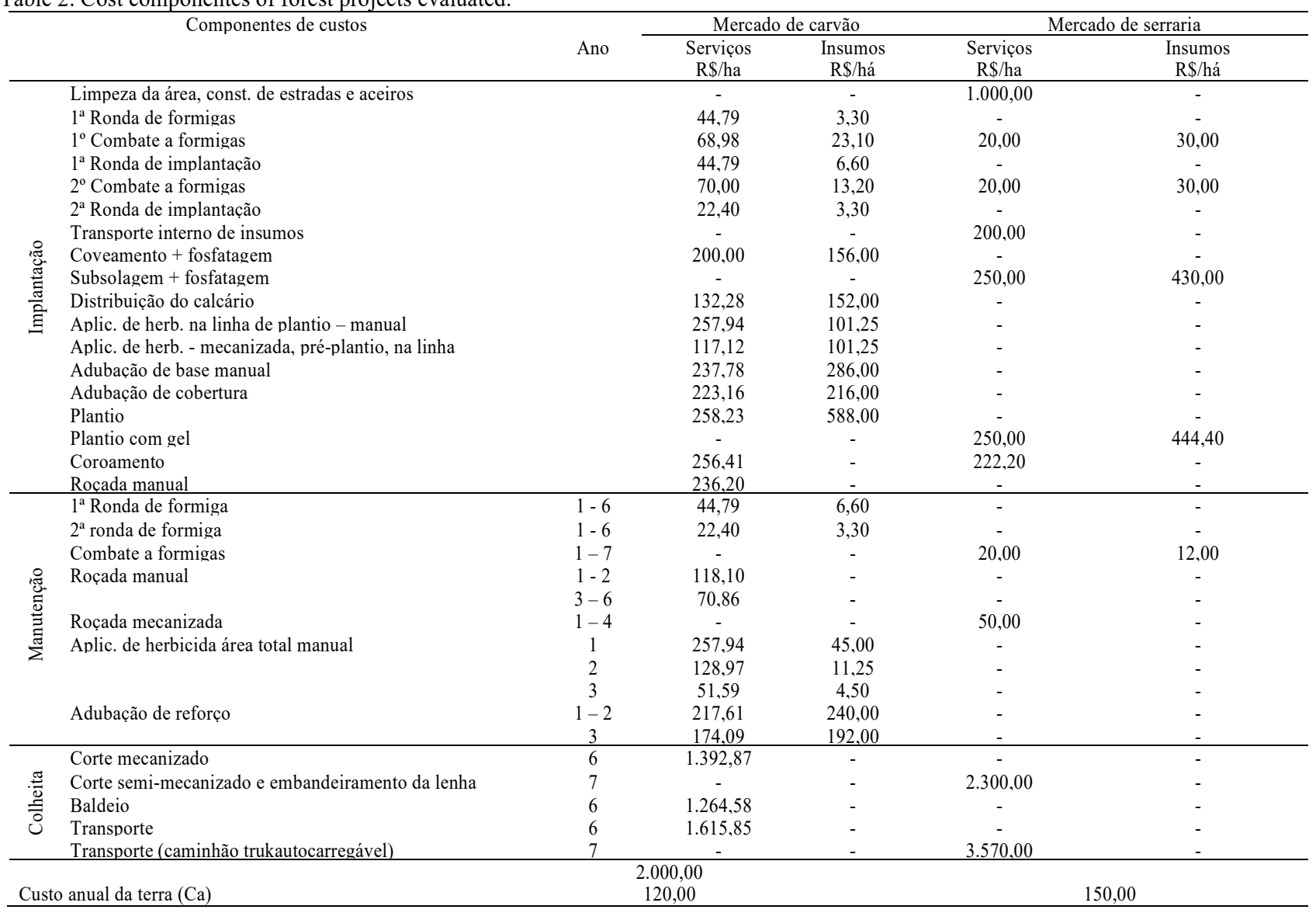

Verificou-se por intermédio da análise de sensibilidade que a elevação na taxa de juros condicionou aumento considerável nos preços mínimos de venda da madeira em todos os cenários avaliados, permitindo inclusive que os respectivos preços alcançassem valores superiores ao de mercado nas regiões de estabelecimentos dos projetos (Figura 3). A Figura 4 ilustra a projeção gráfica para o preço mínimo de venda da madeira em relação à variação na taxa de juros. 
Avaliando a sensibilidade em relação ao custo anual da terra verificou-se que na empresa 1 variação de $10 \%$, para mais, condicionou aumento de $\mathrm{R} \$ 0,33$ no preço mínimo. $\mathrm{Na}$ empresa 2, a mesma alteração proporcionou um aumento de $\mathrm{R} \$ 0,60$ no preço mínimo de venda da madeira (Tabela 4).

Tabela 3. Preço mínimo de venda da madeira nos empreendimentos avaliados.

Table 3. Minimum selling price of timber in the evaluated enterprises.

\begin{tabular}{ccc}
\hline $\begin{array}{c}\text { Formas de } \\
\text { comercialização }\end{array}$ & $\begin{array}{c}\text { Empreendimentos } \\
\text { Florestais }\end{array}$ & $\begin{array}{c}\text { Preço mínimo de } \\
\text { venda }\left(\mathrm{R} \$ / \mathrm{m}^{3}\right)\end{array}$ \\
\hline \multirow{2}{*}{ Madeira em pé } & Empresa 1 & 38,44 \\
& Empresa 2 & 29,76 \\
\hline \multirow{2}{*}{ Madeira empilhada } & Empresa 1 & 55,39 \\
& Empresa 2 & 57,71 \\
\hline
\end{tabular}
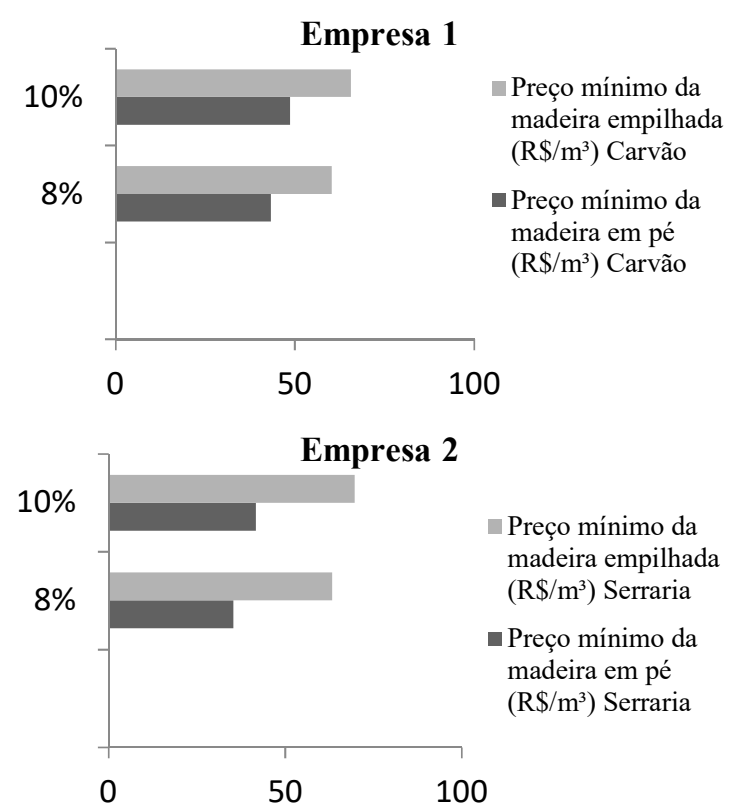

Figura 3. Análise de sensibilidade para o preço mínimo de venda da madeira, com oscilações nas taxas de juros

Fig 3 - Sensitivity analysis for the minimum selling price of the timber considering the interest rate changes.

Obs.: Preço de mercado para a madeira em pé no local de abrangência da empresa $1: \mathrm{R} \$ 39,22 / \mathrm{m}^{3}$

Preço de mercado para a madeira em pé no local de abrangência da empresa $2: \mathrm{R} \$ 32,50 / \mathrm{m}^{3}$

\section{DISCUSSÃO}

Considerando o preço mínimo de venda da madeira em pé, obteve-se um cenário mais atrativo na empresa 2. Isto ocorreu em virtude dos menores custos nos processos de implantação e manutenção (Tabela 3). Em contrapartida, a empresa 2 mostrou maior impacto no custo da colheita, em virtude do baixo nível de mecanização, o que fez com que o preço da madeira empilhada fosse menos atrativa em relação a empresa 1 (Tabela 3). Estudo realizado por Souza; Pires (2009) relata a tendência de redução do custo da colheita florestal pelo processo de mecanização, sobretudo, pelo ganho de produtividade.
Preço mínimo de venda da madeira para a produção de carvão

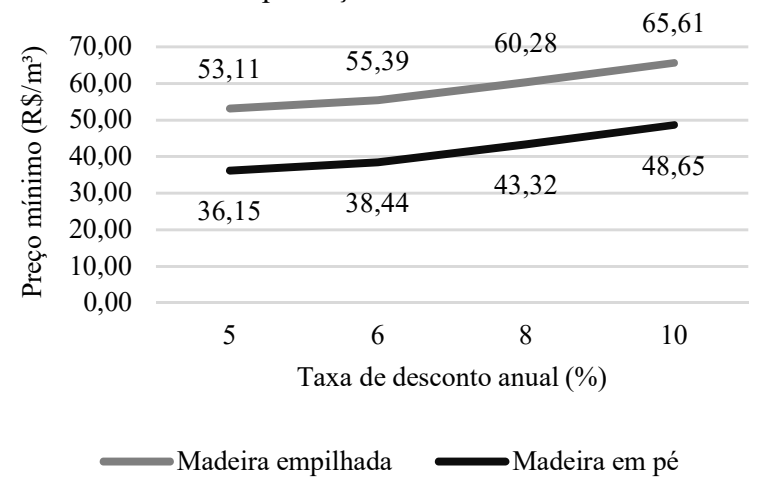

Preço mínimo de venda da madeira para serraria

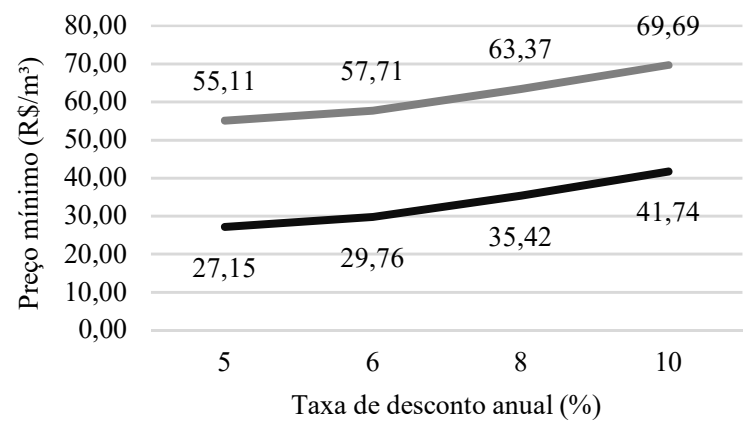

Madeira empilhada $\longrightarrow$ Madeira em pé

Figura 4. Análise de sensibilidade para o preço mínimo de venda da madeira, considerando elevações nas taxas de juros.

Figure 4. Sensitivity analysis for the minimum selling price of timber, considering interest rate increases.

Tabela 4. Análise de sensibilidade para o preço mínimo de venda da madeira, com oscilações do custo anual da terra.

Table 4. Sensitivity analysis for the minimum selling price of the timber considering changes in the annual cost soil.

\begin{tabular}{|c|c|c|c|}
\hline $\begin{array}{c}\text { Situação da } \\
\text { Madeira }\end{array}$ & & $\begin{array}{l}\text { Custo anual da } \\
\text { terra }(\mathrm{Ca})\end{array}$ & $\begin{array}{l}\text { Preço mínimo } \\
\left(\mathrm{R} \$ / \mathrm{m}^{3}\right)\end{array}$ \\
\hline \multirow{10}{*}{$\begin{array}{l}\text { Madeira em } \\
\text { pé }\end{array}$} & \multirow{5}{*}{ Empresa 1} & 108,00 & 38,10 \\
\hline & & 114,00 & 38,27 \\
\hline & & 120,00 & 38,44 \\
\hline & & 126,00 & 38,60 \\
\hline & & 132,00 & 38,77 \\
\hline & \multirow{5}{*}{ Empresa2 } & 135,00 & 29,16 \\
\hline & & 142,50 & 29,46 \\
\hline & & 150,00 & 29,76 \\
\hline & & 157,50 & 30,06 \\
\hline & & 165,00 & 30,36 \\
\hline \multirow{10}{*}{$\begin{array}{l}\text { Madeira } \\
\text { empilhada }\end{array}$} & \multirow{5}{*}{ Empresa 1} & 108,00 & 55,06 \\
\hline & & 114,00 & 55,23 \\
\hline & & 120,00 & 55,39 \\
\hline & & 126,00 & 55,56 \\
\hline & & 132,00 & 55,73 \\
\hline & \multirow{5}{*}{ Empresa2 } & 135,00 & 57,11 \\
\hline & & 142,50 & 57,41 \\
\hline & & 150,00 & 57,71 \\
\hline & & 157,50 & 58,01 \\
\hline & & 165,00 & 58,31 \\
\hline
\end{tabular}


Virgens et al. (2015), utilizando a mesma taxa de juros do presente estudo ( $6 \%$ a.a) e ciclo de corte de sete anos, encontraram valor na ordem de $\mathrm{R} \$ 44,45 / \mathrm{m}^{3}$ para o preço mínimo de venda da madeira em pé. Comparando este valor com aquele observado na pesquisa para a produção de madeira com ciclo de corte de 7 anos (empresa 2), observou redução de $\mathrm{R} \$ 14,69 / \mathrm{m}^{3}$ no preço mínimo. Tal divergência pode ser explicada pela composição diferenciada de custos e receitas ao longo do horizonte de planejamento dos empreendimentos avaliados, o que resulta em diferentes valores para o custo de produção da madeira.

Os valores obtidos para o preço mínimo de venda da madeira não contemplaram os custos de transporte florestal, ou seja, não foi considerada a entrega da madeira no pátio, o que refletiria um impacto significativo nos custos de produção. Pesquisas realizadas por Rezende et al. (2006) relatam o alto impacto do transporte florestal no custo final da matéria prima. Silva et al. (2007) analisando o transporte florestal em um raio de $100 \mathrm{~km}$, com caminhão truck, observaram um impacto no preço mínimo de venda da madeira (custo médio de produção), na ordem de $52,84 \%$. O custo da colheita e transporte responde por um percentual significativo do custo de produção da madeira. Na pesquisa em questão, a colheita proporcionou impacto de $30,60 \%$ e $48,43 \%$ do custo total de produção, respectivamente para as empresas 1 e 2 . Percebe-se, contudo, que se fosse contemplado a entrega da madeira no pátio da indústria (custo do transporte), o preço mínimo da madeira poderia apresentar um aumento de mais de 50\%, conforme relatado por Silva et al. (2007).

Em relação a análise de sensibilidade, observa-se que ao reduzir a taxa de juros o preço mínimo se torna mais atrativo. Taxas mais elevadas de juros impactam a viabilidade dos projetos, favorecendo um cenário de não investimento pelo aumento do preço mínimo de comercialização. Rezende e Oliveira (2001); Soares et al. (2003); Virgens et al. 2016 constataram a relação inversa entre a taxa de juros e a viabilidade dos projetos em relação ao critério Valor Presente Líquido (VPL). De acordo com os mesmos autores tal relação pode ser comprovada pela análise de sensibilidade.

Avaliando a sensibilidade em relação ao custo anual da terra, verificou-se que variações na ordem de $5 \%$ e $10 \%$, para mais e para menos, em ambos os projetos, não influenciaram de forma expressiva na composição do preço mínimo de venda da madeira. Apesar da pequena influência constatada na análise de sensibilidade, o valor da terra assume importância notória no estudo de viabilidade econômica, devendo ser incluído na determinação do custo de produção da madeira (SILVA et al., 2008) de empreendimentos florestais. $\mathrm{O}$ custo da terra deve ser considerado mesmo quando o produtor for o titular da terra, conFigurando neste caso como um custo de oportunidade (SILVA et al., 2005).

Partindo do suposto que quanto menor o preço mínimo de venda da madeira maior será a viabilidade do projeto, pode-se inferir que os empreendimentos florestais terão maiores probabilidades de sucessos em regiões onde a terra apresenta menor grau de especulação ou valorização, considerando, contudo, constante as demais variáveis que possam intervir na viabilidade dos projetos.

Enfim, cabe ressaltar que quaisquer alterações nos custos e receitas dos projetos avaliados implicariam em um novo cenário de preço mínimo para venda da madeira, assim, um adicional de custos em relação às receitas no ano zero (para uma mesma taxa de juros) proporcionaria aumento do preço mínimo, da mesma forma que o aumento das receitas em relação aos custos traria benefícios pela redução do respectivo preço.

\section{CONCLUSÕES}

O preço mínimo de venda da madeira mostrou-se relevante, permitindo nortear o produtor florestal em relação aos reais ganhos quando da comercialização de sua matéria prima, conFigurando assim como uma ferramenta de planejamento econômico para o sucesso dos empreendimentos florestais.

De acordo com a pesquisa, os preços mínimos de venda da madeira apresentaram-se abaixo dos valores de mercado, conFigurando viabilidade aos projetos avaliados.

A análise de sensibilidade contribuiu de forma evidenciar novos cenários para o preço mínimo de venda da madeira frente às variações na taxa de juros e no custo anual da terra.

\section{REFERÊNCIAS}

ALMEIDA, A. N.; SILVA, J. C. G. L; ÂNGELO, H.; BITTENCOURT, A. M.; NUNES, B. E. C. Mercado paranaense de madeira em tora procedente de silvicultura entre 1999 e 2005. Revista Floresta, Curitiba, v. 39, n. 4, p. $869-875,2009$.

ALMEIDA, N. A.; SILVA, J. C. G. L.; ÂNGELO, H.; NUÑEZ, B. E. C. Análise de fatores que influenciam o preço da madeira em tora para processamento mecânico no Paraná. Cerne, Lavras, v. 16, n. 2, p. 243-250, 2010.

IBÁ. Relatório 2017. Indústria Brasileira de Árvores: braziliantreeindustry. Brasília, 2017. 80 p.

KÖPPEN, W. Climatologia: conune studio de los climas de latierra. México: Fondo de Cultura Economica, 1948. 478p.

NOVAES, A. B.; LONGUINHOS, M. A. A.; RODRIGUES, J.; SANTOS, I. F.; GUSMÃO, J. C. Caracterização e demanda florestal da Região Sudoeste da Bahia. In: Memórias do II Simpósio sobre Reflorestamento na Região Sudoeste da Bahia. 1 ed. Colombo: Embrapa Florestas, v. 1, p. 25-43. 2008.

REZENDE, J. L. P.; OLIVEIRA, A. D. Análise Econômica e Social de Projetos Florestais. Viçosa: UFV. 2 ed. 2001. $389 \mathrm{p}$.

REZENDE, J. L. P.; PADUA, C. T. J.; OLIVEIRA, A. D.; SCOLFORO, J. R. S. Análise econômica de fomento florestal com eucalipto no estado de Minas Gerais. Cerne, Lavras, v. 12, n. 3, p. 221-231, 2006.

SILVA, M. L.; JACOVINE, L. A. G.; VALVERDE, S. R. Economia Florestal. 2 ed. Viçosa: UFV, 2005. 1780.

SILVA, M. L.; REZENDE, J. L. P.; LIMA JUNIOR, V. B.; CORDEIRO, S. A.; COELHO JUNIOR, L. M. Métodos de cálculo do custo da terra na atividade florestal. Cerne, Lavras, v. 14. n. 1, p. 75-81. 2008.

SILVA, M. L.; OLIVEIRA, R. J.; VALVERDE, S. R.; MACHADO, C. C.; PIRES, V. A. V. Análise do custo e do raio econômico de transporte de madeira de reflorestamentos para diferentes tipos de veículos. Revista Árvore, Viçosa, v. 31, n. 6, p. 1073-1079, 2007. DOI: http://dx.doi.org/10.1590/S0100-67622007000600012.

SCOLFORO, J. R. S.; JÚNIOR, F. W. A.; OLIVEIRA, A. D.; MAESTRI, R. Simulação e avaliação econômica de regimes de desbastes e desrama para obter madeira de Pinus taeda livre de nós. Ciência Florestal, Santa Maria, v. 11, n. 1, p. 121-139. 2001. 
SOARES, T. S.; CARVALHO, R. M. M. A.; VALE, A. B. Avaliação econômica de um povoamento de Eucalyptus grandis destinado a multiprodutos. Revista Árvore, Viçosa, v. 27, n. 5, p. 689-694, 2003. DOI: http://dx.doi.org/10.1590/S0100-67622003000500011.

VALVERDE, S. R.; SOARES, N. S.; SILVA, M. L.; JACOVINE, L. A. G.; NEIVA, S. A. O comportamento do mercado da madeira de eucalipto no Brasil. Biomassa \& Energia, v. 1, n. 4, p. 393-403, 2004.

VIRGENS, A. P.; FREITAS, L. C.; LEITE, A. M. P. Análise econômica e de sensibilidade em um povoamento implantado no Sudoeste da Bahia. Floresta e Ambiente, Seropédica, v. 23, n. 2, p. 211-219, 2016. DOI: http://dx.doi.org/10.1590/2179-8087.104914.

VIRGENS, A. P.; FREITAS, L. C.; LUZ, D. S.; MOREIRA, A. C. D. Análise econômica e de sensibilidade em projetos de reflorestamentos no estado da Bahia. Enciclopédia Biosfera, Goiânia, v. 11 n. 21, p. 120-127, 2015.

SOUZA, M. A.; PIRES, C. B. Colheita florestal: mensuração e análise dos custos incorridos na atividade mecanizada de extração. Revista Custos e @gronegócio, v. 5, n. 2, p. 104-132, 2009.

VITALE, V.; MIRANDA, G. M. Análise comparativa da viabilidade econômica de plantios de Pinus taeda e Eucalyptus dunnii na região centro sul do Paraná. Revista Floresta, Curitiba, v. 40, n. 3, p. 469-476. 2010. 